\title{
Day Imputed
}

National Cancer Institute

\section{Source}

National Cancer Institute. Day Imputed. NCI Thesaurus. Code C81212.

An imputed date that includes the day of observation. 